\title{
A Generalized of Nörlund Ideal Convergent Double Sequence Spaces
}

\author{
EDUIN RODRÍGUEZ ${ }^{1}$, CARLOS GRANADOS², JUDITH BERMÚDEZ ${ }^{3}$ \\ ${ }^{1}$ Universidad del Atlántico, Barranquilla, COLOMBIA \\ ${ }^{2}$ Universidad de Antioquia, Medellín, COLOMBIA \\ ${ }^{3}$ Universidad del Atlántico, Barranquilla, COLOMBIA
}

Abstract: In this paper, by using the Nörlund mean $N^{t}$ and the notion of ideal double convergence, we introduce new sequence spaces $c_{0}^{\mathcal{I}_{2}}\left(N^{t}\right), c^{\mathcal{I}_{2}}\left(N^{t}\right)$, and $\ell_{\infty}^{\mathcal{I}_{2}}\left(N^{t}\right)$. Besides, we study some topological and algebraic properties on these spaces. Furthermore, some inclusion concerning these spaces are proved.

Key-Words: Nörlund $\mathcal{I}_{2}$-convergence, Nörlund $\mathcal{I}_{2}$-Cauchy, Nörlund $\mathcal{I}_{2}$-bounded.

Received: May 30, 2021. Revised: October 5, 2021. Accepted: October 16, 2021. Published: October 29, 2021.

\section{Introduction and Preliminaries}

Through this paper, $\mathbb{N}$ and $\mathbb{R}$ stand for the sets of natural and real numbers, respectively. Besides, $2^{\omega}=$ $\left\{x=\left(x_{n, m}\right): x \in \mathbb{R}\right.$ (see [17]) denotes the linear space of all real double sequences. Additionally, the sequence spaces $c_{0}, c$, and $\ell_{\infty}$ denote the spaces of all null, convergent, and bounded sequence, respectively, with the usual sup-norm defined by $\mid x \|_{\infty}=$ $\sup _{k}\left|x_{k}\right|$, for each $k \in \mathbb{N}$.

On the other hand, let $\lambda$ and $\mu$ be two double sequence spaces and $A=\left(a_{n k}\right)$ be an infinite matrix of real numbers $a_{n, k}$, where $n, k \in \mathbb{N}$. Then $A$ determines a matrix transformation from $\lambda$ into $\mu$ denoted by $A: \lambda \rightarrow \mu$ such that for every double sequence $x=\left(x_{k, j}\right) \in \lambda$, the double sequence $\{A x=$ $\left.A_{n, m}(x)\right\}$, the $A$-transform of $x$ is in $\mu$. Where the double sequence $\left\{A_{n, m}(x)\right\}$ id defined as:

$$
\left.A_{(} x\right)=\sum_{k=0}^{\infty} a_{n m k} x_{n, m}, \text { for each }(n, m) \in \mathbb{N} \times \mathbb{N}(1)
$$

The pair $(\lambda, \mu)$ stand for the class of all matrices $A$ such that $A$ maps from $\lambda$ into $\mu$. Hence, $A \in(\lambda, \mu)$ if only if the series on the right hand side of (11) converges for each $(n, m) \in \mathbb{N} \times \mathbb{N}$ and every $x \in \lambda$, and we have $A x \in \mu$ for all $x \in \lambda$, where $A_{n, m}=$ $\left(a_{n m k}\right)_{k \in \mathbb{N}}$ express the double sequence in the $(n, m)$ th row of $A$. the matrix domain of an infinite matrix $A$ in a double sequence space $\lambda$ is a double sequence given by

$$
\lambda_{A}:=\left\{x=\left(x_{n, m}\right) \in 2^{\omega}: A x \in \lambda\right\} .
$$

In 2000, Hardy [15] introduced the notion of $\left(t_{k}\right)$ and $T_{n}$ sequences of real number. Now, we will define them on double sequences spaces, let $\left(t_{k, j}\right)$ denote a non-negative double sequence of real numbers and $T_{n, m}=\sum_{k=0}^{n} \sum_{j=0}^{m}, t_{k, j}$, for all $(n, m) \in \mathbb{N}$ with $t_{00}>0$. Then, the Nörlund mean with respect to the double sequence $t=t_{k, j}$ is defined by the matrix $\left(N^{t}\right)=\left(a_{n m k j}^{t}\right)$ as follows:

$\left(a_{n m k j}^{t}\right)= \begin{cases}\frac{t_{n-k, m-j}}{T_{n, m}}, & \text { if } 0 \leq k \leq n \text { and } 0 \leq j \leq m \\ 0, & \text { if } k>n \text { and } j>m\end{cases}$

for all $n, k, m, j \in \mathbb{N}$. It can be proved that the Nörlund matrix $N^{t}$ is regular if and only if $t_{n, m} / T_{n, m} \rightarrow$ 0 as $n, m \rightarrow \infty$.

The double sequence space $\ell_{\infty}\left(N^{t}\right)$ as the set of all sequence whose $N^{t}$-transform are in the space $\ell_{\infty}$ as follows:

$$
\begin{aligned}
& \ell_{\infty}\left(N^{t}\right):=\left\{x=\left(x_{n, m}\right) \in 2^{\omega}:\right. \\
& \left.\sup _{(n, m) \in \mathbb{N} \times \mathbb{N}}\left|\frac{1}{T_{n, m}} \sum_{k=0}^{n} \sum_{j=0}^{m} t_{n-k, m-j} x_{k, j}\right|<\infty\right\} .
\end{aligned}
$$

Moreover, the double sequence spaces $c_{0}\left(N^{t}\right)$ and $c\left(N^{t}\right)$ as the domain of Nörlund mean $N^{t}$ in the spaces $c_{0}$ and $c$, respectively, defined by

$$
\begin{aligned}
& c_{0}\left(N^{t}\right):=\left\{x=\left(x_{n, m}\right) \in 2^{\omega}:\right. \\
& \left.\lim _{n, m \rightarrow \infty} \frac{1}{T_{n, m}} \sum_{k=0}^{n} \sum_{j=0}^{m} t_{n-k, m-j} x_{k, j}=0\right\},
\end{aligned}
$$

and 


$$
\begin{gathered}
c\left(N^{t}\right):=\left\{x=\left(x_{n, m}\right) \in 2^{\omega}: \exists L \in \mathbb{C}\right. \text { such that } \\
\left.\lim _{n, m \rightarrow \infty} \frac{1}{T_{n, m}} \sum_{k=0}^{n} \sum_{j=0}^{m} t_{n-k, m-j} x_{k, j}=L\right\},
\end{gathered}
$$

Taking into account the notions mentioned above, we can easily prove that those spaces are isomorphic to the spaces $c_{0}$ and $c$, respectively. Furthermore, the double sequence $N_{n, m}^{t}(x)$ to denote the $N^{t}$-transform of the double sequence $\left(x_{n, m}\right) \in 2^{\omega}$, where the double sequence $N_{n, m}^{t}(x)$ is given by

$$
\begin{aligned}
& N_{n, m}^{t}(x):=\frac{1}{T_{n, m}} \sum_{k=0}^{n} \sum_{j=0}^{m} t_{n-k, m-j} x_{k, j} \\
& \text { for all }(n, m) \in \mathbb{N} \times \mathbb{N} .
\end{aligned}
$$

Recall in [20] that an ideal $\mathcal{I}$ is a non-empty family of subsets of $\mathbb{N}$ satisfying additive and hereditary properties, that is $(i) \emptyset \in \mathcal{I}$, $(i i)$ for each $A, B \in \mathcal{I}$, we have $A \cup B \in \mathcal{I}$, (iii) for each $A \in \mathcal{I}$ with $B \subseteq A$, we have $B \in \mathcal{I}$. An ideal $\mathcal{I}$ of $\mathbb{N}$ is said to be admissible in $\mathbb{N}$ if and only if $\mathcal{I} \neq \mathbb{N}$ and $\mathcal{I} \supset\{\{n\} n \in \mathbb{N}\}$. A nontrivial ideal $\mathcal{I}$ is said to be maximal if there cannot exist any non-trivial ideal $\mathcal{J}$ containing $\mathcal{I}$ as a subset. A filter $\mathcal{F}$ in $\mathbb{N}$ is a non-empty collection of subsets of $\mathbb{N}$ satisfying $(i) \emptyset \notin \mathcal{F}$, (ii) for each $A, B \in \mathcal{F}$, we have $A \cap B \in \mathcal{F}$, (iii) for each $A \in \mathcal{F}$ with $A \subset B$, we have $B \in \mathcal{F}$. A filter $\mathcal{F}(\mathcal{I})$ which is associated to each ideal $\mathcal{I}$ is given by $\mathcal{F}(\mathcal{I})=\left\{K \subseteq X: K^{c} \in \mathcal{I}\right\}$, where $K^{c}=X \backslash K$. Throughout this paper, $\mathcal{I}_{2}$ is an ideal on $2^{\mathbb{N} \times \mathbb{N}}$ and $\mathcal{F}\left(\mathcal{I}_{2}\right)$ is a filter associated to $\mathcal{I}_{2}$. For more recent notions related to ideal spaces, we refer the reader to [22, 11, 14, 13, 12, 1, 2, 3, 23]. In 2000/2001, Kostyrko et. al [20] generalized the notion of statistical convergence to ideal convergence. Afterwards, Das et al. [4] by using the notions of ideal spaces [20] and double sequences [21] generalized the concept of ideal convergence of double sequence spaces, later Dundar and Altay [5] presented new properties on $\mathcal{I}_{2}$ convergence and $\mathcal{I}_{2}$-Cauchy convergence, then many authors have study those notions in different fields of mathematics $([19,16,6,7,8,9,10])$.

Throughout this paper, $c_{0}^{\mathcal{I}_{2}}, c^{\mathcal{I}_{2}}$, and $\ell_{\infty}^{\mathcal{I}_{2}}$ serve as the $\mathcal{I}_{2}$-null, $\mathcal{I}_{2}$-convergent, and $\mathcal{I}_{2}$-bounded sequence spaces, respectively. In this paper, by using the definitions of the Nörlund mean $N^{t}$, ideal spaces and double convergence, we introduce the notions of Nörlund double sequence spaces $c_{0}^{\mathcal{I}_{2}}\left(N^{t}\right)$, $c^{\mathcal{I}_{2}}\left(N^{t}\right)$, and $\ell_{\infty}^{\mathcal{I}_{2}}\left(N^{t}\right)$ as the sets of all sequences whose $N^{t}$-transform are in the double spaces $c_{0}^{\mathcal{I}_{2}}, c^{\mathcal{I}_{2}}$, and $\ell_{\infty}^{\mathcal{I}_{2}}$ respectively. Furthermore, we study some inclusion relations among these spaces. Besides, we study some topological and algebraic properties on these spaces.

\section{Now, we procure some well-known definitions and results needed for the developing of this paper.}

Definition 1. [4] A double sequence $\left(x_{k j}\right) \in 2^{\omega}$ is said to be $\mathcal{I}_{2}$-convergent to a number $L \in \mathbb{R}$ if, for every $\epsilon>0$, the set $\left\{(k, j) \in \mathbb{N} \times \mathbb{N}:\left|x_{k, j}-L\right| \geq\right.$ $\epsilon\} \in \mathcal{I}_{2}$. And we write $\mathcal{I}_{2}-\lim x_{k, j}=L$. In case $L=0$ then $\left(x_{k, j}\right) \in 2 \omega$ is said to be $\mathcal{I}_{2}$-null.

Definition 2. [5] A sequence $(x)=\left(x_{k, j}\right) \in 2^{\omega}$ is said to be $\mathcal{I}_{2}$-Cauchy if, for every $\epsilon>0$, there exist a numbers $m=m(\epsilon)$ and $n=n(\epsilon)$ such that $\{(k, j) \in$ $\left.\mathbb{N} \times \mathbb{N}:\left|x_{k, j}-x_{m, n}\right| \geq \epsilon\right\} \in \mathcal{I}_{2}$.

Definition 3. [5] A double sequence $\left(x_{k, j}\right) \in 2^{\omega}$ is said to be $\mathcal{I}_{2}$-bounded if there exists $K, j>0$, such that, the set $\left\{(k, j) \in \mathbb{N} \times \mathbb{N}:\left|x_{k, j}\right| \geq K\right\} \in \mathcal{I}_{2}$.

Definition 4. [5] Let $x=\left(x_{k, j}\right)$ and $z=\left(z_{k, j}\right)$ be two double sequences. We say that $x_{k, j}=z_{k, j}$ for almost all $k$ and $J$ relative to $\mathcal{I}_{2}$ (in short a.a.k.r. $\mathcal{I}_{2}$ ) if the set $\{(k, j) \in \mathbb{N} \times \mathbb{N}: x \neq z\} \in \mathcal{I}_{2}$.

Definition 5. [5] A double sequence space $E$ is said to be solid or normal, if $\left(\alpha_{k, j}, x_{k, j}\right) \in E$ whenever $\left(x_{k, j}\right) \in E$ and for any double sequence of scalars $\left(\alpha_{k, j}\right) \in 2 \omega$ with $\left|\alpha_{k, j}\right|<1$, for every $(k, j) \in \mathbb{N} \times \mathbb{N}$.

Lemma 6. [5] Every solid space is monotone.

Definition 7. [5] Let $K=\left\{\left(k_{i}, j_{p}\right) \in \mathbb{N} \times \mathbb{N}\right.$ : $\left.\left(k_{1}, j_{1}\right)<\left(k_{2}, j_{2}\right)<\ldots\right\} \subseteq \mathbb{N} \times \mathbb{N}$ and $E$ be a double sequence space. A $K$-step space of $E$ is a double sequence space $\lambda_{K}^{E}=\left\{\left(x_{k_{i}, j_{p}}\right) \in 2 \omega:\left(x_{k, j}\right) \in E\right\}$.

A canonical pre-image of a double sequence $\left(x_{k_{i}, j_{p}}\right) \in$ is a double sequence $\left(y_{k, j}\right) \in 2 \omega$ defined as follows:

$$
y_{k, j}= \begin{cases}x_{k, j}, & \text { if }(k, j) \in K \\ 0, & \text { otherwise }\end{cases}
$$

A canonical pre-image of a step space $\lambda_{K}^{E}$ is a set of canonical preimages of all elements in $\lambda_{K}^{E}$., i.e., $y$ is in canonical pre-image of $\lambda_{K}^{E}$ iff $y$ is canonical preimage of some element $x \in \lambda_{K}^{E}$.

Definition 8. [5] A double sequence space $E$ is said to be monotone if its contains the canonical preimages of its step space. (i.e., if for all infinite $K \subseteq$ $\mathbb{N} \times \mathbb{N}$ and $\left(x_{k, j}\right) \in E$ the double sequence $\alpha_{k, j} x_{k, j}$, where $\alpha_{k, j}=1$ for $(k, j) \in K$ and $\alpha_{k, j}=0$ otherwise, belongs to $E$ ). 
Definition 9. [5] A double sequence space $E$ is said to be convergence free if $\left(x_{k, j}\right) \in E$, whenever $\left(y_{k, j}\right) \in E$ and $\left(y_{k, j}\right)=0$ imply that $\left(x_{k, j}\right)=0$ for all $(k, j) \in \mathbb{N} \times \mathbb{N}$.

Definition 10. $[18]$ A map $h$ defined on a domain $D \subset X$ i.e., $h: \bar{D} \subset X \rightarrow \mathbb{R}$ is said to satisfy Lipschitz condition if $|h(x)-h(y)| \leq K|x-y|$, where $K$ is known as the Lipschitz constant.

Remark 11. [5] A convergence field of $\mathcal{I}_{2}$ convergence is a set

$$
\mathcal{F}\left(\mathcal{I}_{2}\right)=\left\{x=\left(x_{k, j}\right) \in \ell_{\infty}: \text { there exists } \mathcal{I}_{2}-\lim x \in \mathbb{R}\right\}
$$

Definition 12. [20] The convergence field $\mathcal{F}\left(\mathcal{I}_{2}\right)$ is a closed linear subspace of $\ell_{\infty}$ with respect to the supremum norm, $\mathcal{F}\left(\mathcal{I}_{2}\right)=\ell_{\infty} \cap c^{\mathcal{I}_{2}}$.

Lemma 13. [5] Let $K \in \mathcal{F}\left(\mathcal{I}_{2}\right)$ and $M \subseteq \mathbb{N} \times \mathbb{N}$. If $M \notin \mathcal{I}_{2}$, then $M \cap K \notin \mathcal{I}_{2}$.

Definition 14. [18] The function $h: D \subset X \rightarrow \mathbb{R}$ defined by $h(x)=\mathcal{I}_{2}-\lim x$, for all $x \in \mathcal{F}\left(\mathcal{I}_{2}\right)$ is a Lipschitz function.

\section{Main Results}

In this section, we introduce the notions of the sequence spaces $c_{0}^{\mathcal{I}_{2}}\left(N^{t}\right), c^{\mathcal{I}_{2}}\left(N^{t}\right)$, and $\ell_{\infty}^{\mathcal{I}_{2}}\left(N^{t}\right)$ as the sets of all double sequences whose $N^{t}$-transforms are in the spaces $c_{0}^{\mathcal{I}_{2}}, c^{\mathcal{I}_{2}}$, and $\ell_{\infty}^{\mathcal{I}_{2}}$ respectively. Moreover, we study some inclusion relation topological and algebraic properties on these spaces. Throughout the article, we suppose that the double sequences $x=\left(x_{k, j}\right) \in 2^{\omega}$ and $N_{n, m}^{t}(x)$ are connected with the relations defined in the last section and $\mathcal{I}_{2}$ is strongly admissible ideal of subset of $\mathbb{N} \times \mathbb{N}$. Define,

$$
\begin{aligned}
& c_{0}^{\mathcal{I}_{2}}\left(N^{t}\right):=\left\{x=\left(x_{k, j}\right) \in 2^{\omega}:\right. \\
& \left.\left\{(n, m) \in \mathbb{N} \times \mathbb{N}:\left|N_{n, m}^{t}(x)\right| \geq \epsilon\right\} \in \mathcal{I}_{2}\right\},
\end{aligned}
$$

and

$$
\begin{aligned}
& c^{\mathcal{I}_{2}}\left(N^{t}\right):=\left\{x=\left(x_{k, j}\right) \in 2^{\omega}:\{(n, m) \in \mathbb{N} \times \mathbb{N}:\right. \\
& \left.\left.\left|N_{n, m}^{t}(x)-L\right| \geq \epsilon \text { for some } L \in \mathbb{R}\right\} \in \mathcal{I}_{2}\right\},
\end{aligned}
$$

$$
\begin{aligned}
& \ell_{\infty}^{\mathcal{I}_{2}}\left(N^{t}\right):=\left\{x=\left(x_{k, j}\right) \in 2^{\omega}: \exists K>0\right. \text { s.t } \\
& \left.\left\{(n, m) \in \mathbb{N} \times \mathbb{N}:\left|N_{n, m}^{t}(x)\right| \geq K\right\} \in \mathcal{I}_{2}\right\} .
\end{aligned}
$$

We write

$$
m_{0}^{\mathcal{I}_{2}}\left(N^{t}\right):=c_{0}^{\mathcal{I}_{2}}\left(N^{t}\right) \cap \ell_{\infty}\left(N^{t}\right),
$$

and

$$
m^{\mathcal{I}_{2}}\left(N^{t}\right):=c^{\mathcal{I}_{2}}\left(N^{t}\right) \cap \ell_{\infty}\left(N^{t}\right) .
$$

With the notation of (2), the spaces $c_{0}^{\mathcal{I}_{2}}\left(N^{t}\right), c^{\mathcal{I}_{2}}\left(N^{t}\right), \ell_{\infty}^{\mathcal{I}_{2}}\left(N^{t}\right), m^{\mathcal{I}_{2}}\left(N^{t}\right)$ and $m_{0}^{\mathcal{I}_{2}}\left(N^{t}\right)$ can be redefined as follows:

$$
\begin{aligned}
& c_{0}^{\mathcal{I}_{2}}\left(N^{t}\right)=\left(c_{0}^{\mathcal{I}_{2}}\right)_{N^{t}}, \\
& c^{\mathcal{I}_{2}}\left(N^{t}\right)=\left(c^{\mathcal{I}_{2}}\right)_{N^{t}}, \\
& \ell_{\infty}^{\mathcal{I}_{2}}\left(N^{t}\right)=\left(\ell_{\infty}^{\mathcal{I}_{2}}\right)_{N^{t}}, \\
& m^{\mathcal{I}_{2}}\left(N^{t}\right)=\left(m^{\mathcal{I}_{2}}\right)_{N^{t}} \\
& m_{0}^{\mathcal{I}_{2}}\left(N^{t}\right)=\left(m_{0}^{\mathcal{I}_{2}}\right)_{N^{t}} .
\end{aligned}
$$

Definition 15. Let $I_{2}$ be a strongly admissible ideal of subset of $\mathbb{N} \times \mathbb{N}$. If for each $\epsilon>0$ there exist numbers $s=s(\epsilon) \in \mathbb{N}$ and $p=p(\epsilon) \in \mathbb{N}$ such that $\{(n, m) \in$ $\left.\mathbb{N} \times \mathbb{N}:\left|N_{n, m}^{t}(x)-N_{s, p}^{t}(x)\right| \geq \epsilon\right\} \in \mathcal{I}_{2}$, then $a$ double sequence $(x)=\left(x_{k, j}\right) \in 2^{\omega}$ is called Nörlund $\mathcal{I}_{2}$-Cauchy.

Example 16. Define $\mathcal{I}_{2_{f}}=\{A \subseteq \mathbb{N} \times \mathbb{N}$ : $A$ is finite $\}. \quad \mathcal{I}_{2_{f}}$ is a strongly admissible ideal in $\mathbb{N} \times \mathbb{N}$ and $c^{\mathcal{I}_{2_{f}}}\left(N^{t}\right)=c\left(N^{t}\right)$.

Example 17. Consider a non-trivial ideal $\mathcal{I}_{2_{d}}=$ $\{A \subseteq \mathbb{N} \times \mathbb{N}: d(A)=0$, where $d(A)$ denotes the double natural density of the set $A$. In this case, $c^{\mathcal{I}_{2}}\left(N^{t}\right)=S\left(N^{t}\right)$, where $S\left(N^{t}\right)$ is the space of all Nörlund statistically convergent double sequence defined as:

$$
\begin{aligned}
& S\left(N^{t}\right):=\left\{x=\left(x_{k, j}\right) \in 2^{\omega}:\right. \\
& d\left(\left\{(n, m) \in \mathbb{N} \times \mathbb{N}:\left|N_{n, m}^{t}(x)-L\right| \geq \epsilon\right\}\right)=0,
\end{aligned}
$$

for some $L \in \mathbb{R}\}$.

Theorem 18. The double sequence spaces $c_{0}^{\mathcal{I}_{2}}\left(N^{t}\right), c^{\mathcal{I}_{2}}\left(N^{t}\right), \ell_{\infty}^{\mathcal{I}_{2}}\left(N^{t}\right), m^{\mathcal{I}_{2}}\left(N^{t}\right)$ and $m_{0}^{\mathcal{I}_{2}}\left(N^{t}\right)$ are real vector spaces.

Proof. Let $x=\left(x_{k, j}\right), y=y_{k, j}$ be two arbitrary elements of the space $c^{\mathcal{I}_{2}}\left(N^{t}\right)$ and $\alpha, \beta$ are scalars. Now, since $x, y \in c^{\mathcal{I}_{2}}\left(N^{t}\right)$, then for given $\epsilon>0$, there exist $L_{1}, L_{2} \in \mathbb{R}$, such that

$$
\left\{(n, m) \in \mathbb{N} \times \mathbb{N}:\left|N_{n, m}^{t}(x)-L_{1}\right| \geq \frac{\epsilon}{2}\right\} \in \mathcal{I}_{2},
$$

and

$$
\left\{(n, m) \in \mathbb{N} \times \mathbb{N}:\left|N_{n, m}^{t}(y)-L_{2}\right| \geq \frac{\epsilon}{2}\right\} \in \mathcal{I}_{2} .
$$

Now, let

$$
A_{1}=\left\{(n, m) \in \mathbb{N} \times \mathbb{N}:\left|N_{n, m}^{t}(x)-L_{1}\right|<\frac{\epsilon}{2|\alpha|}\right\} \in \mathcal{F}\left(\mathcal{I}_{2}\right),
$$


$A_{2}=\left\{(n, m) \in \mathbb{N} \times \mathbb{N}:\left|N_{n, m}^{t}(y)-L_{2}\right|<\frac{\epsilon}{2|\beta|}\right\} \in \mathcal{F}\left(\mathcal{I}_{2}\right)$, be such that $A_{1}^{c}, A_{2}^{c} \in \mathcal{I}_{2}$. Then

$$
\begin{aligned}
& A_{3}=\{(n, m) \in \mathbb{N} \times \mathbb{N}: \\
& \left|\alpha N_{n, m}^{t}(x)+\beta N_{n, m}^{t}(y)-\left(\alpha L_{1}+\beta L_{2}\right)\right| \\
& <\epsilon\} \supseteq\left\{A_{1} \cap A_{2}\right\}
\end{aligned}
$$

Thus, the sets on the right hand sides of (10) belong to $\mathcal{F}\left(\mathcal{I}_{2}\right)$. By the definition of filter associated with an ideal $\mathcal{I}_{2}$, the complement of the set on the left hand side of $(10)$ belongs to $\mathcal{I}_{2}$. This implies that $(\alpha x+$ $\beta y) \in c^{\mathcal{I}_{2}}\left(N^{t}\right)$. Hence, $c^{\mathcal{I}_{2}}\left(N^{t}\right)$ is a linear space.

The proof of the remaining results are similar.

Theorem 19. Let $\mathcal{I}_{2} \subseteq 2^{\mathbb{N} \times \mathbb{N}}$ be a non-trivial ideal. Then the inclusion $c\left(N^{t}\right) \subset c^{\mathcal{I}_{2}}\left(N^{t}\right)$ is strict.

Proof. The inclusion $c \subseteq c^{\mathcal{I}_{2}}$ is obvious and for any $X$ and $Y$ spaces, if $X \subseteq Y$, then $X\left(N^{t}\right) \subseteq$ $Y\left(N^{t}\right)$. Therefore, we have $c\left(N^{t}\right) \subset c^{\mathcal{I}_{2}}\left(N^{t}\right)$. For strict inclusion consider the following example.

Example 20. Define the double sequence $x=$ $\left(x_{k, j}\right) \in 2^{\omega}$ such that

$$
N_{n, m}^{t}= \begin{cases}\sqrt{n m}, & \text { if } m n=(i j)^{2}, \text { for }(i, j) \in \mathbb{N} \times \mathbb{N} \\ n m, & \text { otherwise. }\end{cases}
$$

Then $x \in c_{d}^{\mathcal{I}_{2}}\left(N^{t}\right)$ but $x \notin c\left(N^{t}\right)$.

Theorem 21. A double sequence $x=\left(x_{k, j}\right) \in 2 \omega$ is Nörlund $\mathcal{I}_{2}$-convergent if only if for every $\epsilon$, there exist $s=s(\epsilon) \in \mathbb{N}$ and $p=p(\epsilon) \in \mathbb{N}$, such that

$$
\left\{(n, m) \in \mathbb{N} \times \mathbb{N}:\left|N_{n, m}^{t}(x)-N_{s, p}^{t}(x)\right|<\epsilon\right\} \in \mathcal{F}\left(\mathcal{I}_{2}\right) .
$$

Proof. Let the double sequence $x=\left(x_{n, m}\right) \in 2 \omega$ be Nörlund $\mathcal{I}_{2}$-convergent to some number $L \in \mathbb{R}$. Then, for given $\epsilon>0$, the set

$$
A_{\epsilon}=\left\{(n, m) \in \mathbb{N} \times \mathbb{N}:\left|N_{n, m}^{t}(x)-L\right|<\frac{\epsilon}{2}\right\} \in \mathcal{F}\left(\mathcal{I}_{2}\right) .
$$
have

Fix a integers $s=s(\epsilon), p=p(\epsilon) \in A_{\epsilon}$. Then, we

$$
\begin{aligned}
& \left|N_{n, m}^{t}(x)-N_{s, p}^{t}(x)\right| \leq \\
& \left|N_{n, m}^{t}(x)-L\right|+\left|L-N_{s, p}^{t}(x)\right| \\
& <\frac{\epsilon}{2}+\frac{\epsilon}{2}=\epsilon
\end{aligned}
$$

for all $n, m \in A_{\epsilon}$. Hence (11) holds. Then

$$
\begin{aligned}
& E_{\epsilon}=\{(n, m) \in \mathbb{N} \times \mathbb{N}: \\
& N_{n, m}^{t}(x) \in\left[N_{n, m}^{t}(x)-\epsilon, N_{s, p}^{t}(x)+\epsilon\right] \\
& \in \mathcal{F}\left(\mathcal{I}_{2}\right), \text { for all } \epsilon>0 .
\end{aligned}
$$

Let $J_{\epsilon}=\left[N_{n, m}^{t}(x)-\epsilon, N_{s, p}^{t}(x)+\epsilon\right]$. Fixing $\epsilon>0$, we have $E_{\epsilon} \in \mathcal{F}\left(\mathcal{I}_{2}\right)$ as well as $E_{\frac{\epsilon}{2}} \in \mathcal{F}\left(\mathcal{I}_{2}\right)$. Hence $E_{\epsilon} \cap E_{\frac{\epsilon}{2}} \in \mathcal{F}\left(\mathcal{I}_{2}\right)$. This implies that $J=J_{\epsilon} \cap J_{\frac{\epsilon}{2}} \neq \emptyset$. That is, $\left\{(n, m) \in \mathbb{N} \times \mathbb{N}: N_{n, m}^{t}(x) \in J\right\} \in \mathcal{F}\left(\mathcal{I}_{2}\right)$ and thus, $\operatorname{diam}(J) \leq \frac{1}{2} \operatorname{diam}\left(J_{\epsilon}\right)$, where, the diam of $J$ denotes the length of interval $J$. Proceeding in this way, by induction, we get a double sequence of closed intervals

$$
J_{\epsilon}=\mathcal{I}_{0,0} \supseteq \mathcal{I}_{1,1} \supseteq \cdots \supseteq \mathcal{I}_{n, m} \supseteq \cdots
$$

Such that

$\operatorname{diam}\left(\mathcal{I}_{n, m}\right) \leq \frac{1}{2} \operatorname{diam}\left(\mathcal{I}_{n-1, m-1}\right)$ for $n, m=(2,3, \ldots)$ and

$$
\left\{(n, m) \in \mathbb{N} \times \mathbb{N}: N_{n, m}^{t}(x) \in \mathcal{I}_{n, m}\right\} \in \mathcal{F}\left(\mathcal{I}_{2}\right) .
$$

Then there exists a number $L \in \cap_{(n, m) \in \mathbb{N} \times \mathbb{N}}$ and it is a routine work to verify that $L=\mathcal{I}_{2}-\lim N_{n, m}^{t}(x)$ showing that $x=\left(x_{k, j}\right) \in 2 \omega$ is Nörlund $\mathcal{I}_{2}$ converges.

Theorem 22. The inclusions $c_{0}^{\mathcal{I}_{\in}}\left(N^{t}\right) \subset c^{\mathcal{I}_{2}}\left(N^{t}\right) \subset$ $\ell_{\infty}^{\mathcal{I}_{2}}\left(N^{t}\right)$ are strict.

Proof. The inclusion $c_{0}^{\mathcal{I}_{2}}\left(N^{t}\right) \subset c^{\mathcal{I}_{2}}\left(N^{t}\right)$ is obvious. Now, to show the strictness of the inclusion, consider the double sequence $x=\left(x_{k, j}\right) \in 2 \omega$ such that $N_{n, m}^{t}(x)=1$. It is easy to see that the double sequence $N_{n, m}^{t}(x) \in c^{\mathcal{I}_{2}}$ but $N_{n, m}^{t}(x) \notin$ $c_{0}^{\mathcal{I}_{2}}$. That is, $x \in c^{\mathcal{I}_{2}}\left(N^{t}\right) \backslash c_{0}^{\mathcal{I}_{2}}\left(N^{t}\right)$. Next, let $x=\left(x_{k, j}\right) \in c^{\mathcal{I}_{2}}\left(N^{t}\right)$. Then there exists a number $L \in \mathbb{R}$ such that $\mathcal{I}_{2}$ - $\lim \left|N_{n, m}^{t}(x)-L\right|=0$. That is $\left\{(n, m) \in \mathbb{N} \times \mathbb{N}:\left|N_{n, m}^{t}(x)-L\right| \geq \epsilon\right\} \in \mathcal{I}_{2}$. We have

$\left|N_{n, m}^{t}(x)\right|=\left|N_{n, m}^{t}(x)-L+L\right| \leq\left|N_{n, m}^{t}(x)-L\right|+|L|$.

From this it easily follows that the double sequence $\left(x_{k, j}\right)$ must belong to $\ell_{\infty}^{\mathcal{I}_{2}}\left(N^{t}\right)$. Further, we show the strictness of the inclusion $c^{\mathcal{I}_{2}}\left(N^{t}\right) \subset \ell_{\infty}^{\mathcal{I}_{2}}\left(N^{t}\right)$ by constructing the following example.

Example 23. Consider the double sequence $x=$ $\left(x_{k, j}\right) \in 2 \omega$ to be such that

$$
N_{n, m}^{t}(x)= \begin{cases}\sqrt{n m}, & \text { if } n m \text { is square } \\ 1, & \text { if } n m \text { is odd non-square } \\ 0, & \text { if } n m \text { is even non-square. }\end{cases}
$$


Then the double sequence $N_{n, m}^{t}(x) \in \ell_{\infty}^{\mathcal{I}_{2}}$ but $N_{n, m}^{t}(x) \notin c^{\mathcal{I}_{2}}$, which implies that the double sequence $x \in \ell_{\infty}^{\mathcal{I}_{2}}\left(N^{t}\right) \backslash c^{\mathcal{I}_{2}}\left(N^{t}\right)$. Thus, the inclusion $c_{0}^{\mathcal{I}_{2}}\left(N^{t}\right) \subset c^{\mathcal{I}_{2}}\left(N^{t}\right) \subset \ell_{\infty}^{\mathcal{I}_{2}}\left(N^{t}\right)$ are strict.

Remark 24. A Nörlund bounded double sequence is obviously Nörlund $\mathcal{I}_{2}$-bounded as the empty set belongs to the ideal $\mathcal{I}_{2}$. However, the converse is not true. For example, we consider the double sequence

$$
N_{n, m}^{t}(x)= \begin{cases}\sqrt{n m}, & \text { if } n m \text { is square } \\ 0, & \text { if } n m \text { is not square. }\end{cases}
$$

Clearly $N_{n, m}^{t}(x)$ is not a bounded double sequence. However, $\left\{(n, m) \in \mathbb{N} \times \mathbb{N}\left|N_{n, m}^{t}(x)\right| \geq \frac{1}{2}\right\} \in \mathcal{I}_{2}$. Hence $\left(x_{k, j}\right)$ is Nörlund $\mathcal{I}_{2}$-bounded.

Theorem 25. The spaces $m^{\mathcal{I}_{2}}\left(N^{t}\right)$ and $m_{0}^{\mathcal{I}_{2}}\left(N^{t}\right)$ are Banach spaces normed by

$\|x\|_{X\left(N^{t}\right)}=\sup _{n, m}\left|N_{n, m}^{t}(x)\right|$ where $X \in\left\{m^{\mathcal{I}_{2}}, m_{0}^{\mathcal{I}_{2}}\right\}$.

Proof. Let $\left(x_{k, j}^{i, p}\right)$ be a Cauchy sequence in $m^{\mathcal{I}_{2}}\left(N^{t}\right) \subset \ell_{\infty}\left(N^{t}\right)$. Then we have $\left(x_{k, j}^{i, p}\right)$ converges in $\ell_{\infty}\left(N^{t}\right)$ and $\lim _{i, p \rightarrow \infty} N_{n, m}^{t}\left(x^{i, p}\right)=N_{n, m}^{t}(x)$. Let $\mathcal{I}_{2}-\lim N_{n, m}^{t}\left(x^{i, p}\right)=\ell_{i, p}$ for each $(i, p) \in \mathbb{N} \times \mathbb{N}$. Then we have to show that

(i) $\left(L_{i, p}\right)$ is convergent say to $L$,

(ii) $\mathcal{I}_{2}-\lim N_{n, m}^{t}(x)=L$.

(i) Since $\left(x_{k, j}\right)$ is Cauchy, so for each $\epsilon>0$ there exist $\left(n_{0}, m_{0}\right) \in \mathbb{N} \times \mathbb{N}$ such that

$$
\left|N_{n, m}^{t}\left(x^{i, p}\right)-N_{n, m}^{t}\left(x^{q, w}\right)\right|<\frac{\epsilon}{3},
$$

for all $q \geq n_{0}$ and $w \geq m_{0}$

Now, let $A$ and $B$ be the following sets in $\mathcal{I}_{2}$ :

$$
A=\left\{(n, m) \in \mathbb{N} \times \mathbb{N}:\left|N_{n, m}^{t}\left(x^{i, p}\right)-L_{i, p}\right| \geq \frac{\epsilon}{3}\right\}
$$

and

$$
B=\left\{(n, m) \in \mathbb{N} \times \mathbb{N}:\left|N_{n, m}^{t}\left(x^{i, p}\right)-L_{q, w}\right| \geq \frac{\epsilon}{3}\right\}
$$

Consider $i, q \geq n_{0} ; p, w \geq m_{0}$ and $(n, m) \notin A \cap B$. Then we have

$\left|L_{i, p}-L_{q, w}\right| \leq\left|N_{n, m}^{t}\left(x^{i, p}\right)-L_{i, p}\right|+$ $\left|N_{n, m}^{t}\left(x^{i, p}\right)-L_{q, w}\right|$

$\left|N_{n, m}^{t}\left(x^{i, p}\right)-N_{n, m}^{t}\left(x^{q, w}\right)\right|<\epsilon$ by (12), (13), (14). Thus $\left(L_{i, p}\right)$ is a Cauchy sequence of $\mathbb{R}$ and thus convergent, say to $L$, that is, $\lim _{i, p \rightarrow \infty} L_{i, p}=L$. (ii) Let $\delta>0$ be given, then we can fined $u_{0}$ and $v_{0}$ such that

$$
\left|L_{i, p}-L\right|<\frac{\delta}{3} \text { for each } i>u_{0} \text { and } p>v_{0}
$$

We have $\left(x_{k, j}^{q, w}\right) \rightarrow x_{k, j}$ as $i, p \rightarrow \infty$. Thus

$$
\left|N_{n, m}^{t}\left(x^{i, p}\right)-N_{n, m}^{t}(x)\right|<\frac{\delta}{3}
$$

for each $i>u_{0}$ and $p>v_{0}$

Since $\left(x_{k, j}^{q, w}\right)$ is $\mathcal{I}_{2}$-convergent to $L_{q, w}$, there exists $D \in \mathcal{I}_{2}$ such that, for each $(n, m) \notin D$, we have

$$
\left|N_{n, m}^{t}\left(x^{q, w}\right)-L_{q, w}\right|<\frac{\delta}{3}
$$

Without loss of generality, let $q>u_{0} ; w>v_{0}$ then for all $(n, m) \notin D$, we have by (15),$(16)$, and (17) that

$$
\begin{aligned}
& \left|N_{n, m}^{t}(x)-L\right| \leq \\
& \left|N_{n, m}^{t}(x)-N_{n, m}^{(t)}\left(x^{q, w}\right)\right|+\left|N_{n, m}^{(t)}\left(x^{q, w}\right)-L_{q, w}\right| \\
& +\left|L_{q, w}-L\right|<\delta .
\end{aligned}
$$

Hence $\left(x_{k, j}\right)$ is Nörlund $\mathcal{I}_{2}$-convergent to $L$. Thus $m^{\mathcal{I}_{2}}\left(N^{t}\right)$ is a Banach space. The other cases can be similarly proved.

Theorem 26. The spaces $c_{0}^{\mathcal{I}_{2}}\left(N^{t}\right)$ and $m_{0}^{\mathcal{I}_{2}}\left(N^{t}\right)$ are solid and monotone.

Proof. We shall prove the result for $c_{0}^{\mathcal{I}_{2}}\left(N^{t}\right)$. Other follows similarity. Let $x=\left(x_{k, j}\right) \in c_{0}^{\mathcal{I}_{2}}\left(N^{t}\right)$. for $\epsilon>0$, the set

$$
\left\{(n, m) \in \mathbb{N} \times \mathbb{N}:\left|N_{n, m}^{t}(x)\right| \geq \epsilon\right\} \in \mathcal{I}_{2} .
$$

Let $\alpha=\left(\alpha_{k, j}\right)$ be a double sequence of scalars with $|\alpha| \leq 1$ for all $(k, j) \in \mathbb{N} \times \mathbb{N}$. Then

$$
\begin{aligned}
& \left|N_{n, m}^{t}(\alpha x)\right| \\
& =\left|\alpha N_{n, m}^{t}(x)\right| \\
& \leq|\alpha|\left|N_{n, m}^{t}(x)\right| \\
& \leq\left|N_{n, m}^{t}(x)\right|, \\
& \text { for all }(n, m) \in \mathbb{N} \times \mathbb{N} .
\end{aligned}
$$

Thus, from the above inequality and (18):

$$
\begin{aligned}
& \left\{(n, m) \in \mathbb{N} \times \mathbb{N}:\left|N_{n, m}^{t}(\alpha x)\right|\right. \\
& \geq \epsilon \subseteq\{(n, m) \in \mathbb{N} \times \mathbb{N}: \\
& \left|N_{n, m}^{t}(x)\right| \geq \epsilon \in \mathcal{I}_{2}
\end{aligned}
$$


implies $\left\{(n, m) \in \mathbb{N} \times \mathbb{N}:\left|N_{n, m}^{t}(\alpha x)\right| \geq \epsilon\right\} \quad \epsilon$ $\mathcal{I}_{2}$. . Therefore, $\left(\alpha x_{n, m}\right) \in c_{0}^{\mathcal{I}_{2}}\left(N^{t}\right)$. Hence the space $c_{0}^{\mathcal{I}_{2}}$ is solid, and hence by Lemma $(6)$ the space $c_{0}^{\mathcal{I}_{2}}\left(N^{t}\right)$ is monotone.

Theorem 27. The spaces $c^{\mathcal{I}_{2}}\left(N^{t}\right)$ and $c_{0}^{\mathcal{I}_{2}}\left(N^{t}\right)$ are not convergence free.

Proof. The proof of this result follows from the following example.

Example 28. Let $\mathcal{I}_{2}=\mathcal{I}_{2_{d}}$. Consider $x_{k, j}=\frac{1}{k j}$ and $y_{k, j}=k j$ for all $k, j$. Then $\left(x_{k, j}\right)$ belongs to $c^{\mathcal{I}_{2}}\left(N^{t}\right)$ and $c_{0}^{\mathcal{I}_{2}}\left(N^{t}\right)$, but $\left(y_{k, j}\right)$ does not belongs to $c^{\mathcal{I}_{2}}\left(N^{t}\right)$ and $c_{0}^{\mathcal{I}_{2}}\left(N^{t}\right)$. Hence, the spaces are not convergence free.

Theorem 29. The function $g: m^{\mathcal{I}_{2}}\left(N^{t}\right) \rightarrow \mathbb{R}$ defined by $g(x)=\left|\mathcal{I}_{2}-\lim N_{n, m}^{t}(x)\right|$, where $m^{\mathcal{I}_{2}}\left(N^{t}\right)=$ $\ell_{\infty}\left(N^{t}\right) \cap c^{\mathcal{I}_{2}}\left(N^{t}\right)$, is Lipschitz function and hence uniformly continuous.

Proof. First of all, we show that the function is well defined. Let $x, y \in m_{2}^{\mathcal{I}}\left(N^{t}\right)$, such that

$$
\begin{aligned}
& x=y \\
& \Rightarrow \mathcal{I}_{2}-\lim N_{n, m}^{t}(x)=\mathcal{I}_{2}-\lim N_{n, m}^{t}(y) \\
& \Rightarrow\left|\mathcal{I}_{2}-\lim N_{n, m}^{t}(x)\right|=\left|\mathcal{I}_{2}-\lim N_{n, m}^{t}(y)\right| \\
& \Rightarrow g(x)=g(y) .
\end{aligned}
$$

Thus, $g$ is well defined. Next, let $x=\left(x_{k, j}\right), y=$ $y_{k, j} \in m^{\mathcal{I}_{2}}\left(N^{t}\right), x \neq y$. Then

$$
\begin{aligned}
& A_{1}=\{(n, m) \in \mathbb{N} \times \mathbb{N}: \\
& \left|N_{n, m}^{t}(x)-g(x)\right| \geq|x-y|_{*} \in \mathcal{I}_{2}, \\
& A_{2}=\{(n, m) \in \mathbb{N} \times \mathbb{N}: \\
& \left|N_{n, m}^{t}(y)-g(y)\right| \geq|x-y|_{*} \in \mathcal{I}_{2},
\end{aligned}
$$

where $|x-y|_{*}=\sup _{n, m}\left|N_{n, m}^{t}(x)-N_{n, m}^{t}(y)\right|$. Thus

$$
\begin{aligned}
& B_{1}=\{(n, m) \in \mathbb{N} \times \mathbb{N}: \\
& \left|N_{n, m}^{t}(x)-g(x)\right|<|x-y|_{*} \in \mathcal{F}\left(\mathcal{I}_{2}\right)
\end{aligned}
$$

and

$$
\begin{aligned}
& B_{2}=\{(n, m) \in \mathbb{N} \times \mathbb{N}: \\
& \left|N_{n, m}^{t}(y)-g(y)\right|<|x-y|_{*} \in \mathcal{F}\left(\mathcal{I}_{2}\right) .
\end{aligned}
$$

Hence $B=B_{1} \cap B_{2} \in \mathcal{F}\left(\mathcal{I}_{2}\right)$, so that $B$ is non-empty set. Therefore choosing $(n, m) \in B$, we have

$$
\begin{aligned}
& |g(x)-g(y)| \leq\left|g(x)-N_{n, m}^{t}(x)\right|+ \\
& \left|N_{n, m}^{t}(x)-N_{n, m}^{t}(y)\right|+\left|N_{n, m}^{t}(y)-g(y)\right| \\
& \leq 3|x-y|_{*}
\end{aligned}
$$

Thus, $g$ is Lipschitz function and hence uniformly continuous.

Theorem 30. If $x=\left(x_{k, j}\right), y=\left(y_{k, j}\right) \in m^{\mathcal{I}_{2}}\left(N^{t}\right)$ with $N^{t}(x \cdot y)=N^{t}(x) \cdot N^{t}(y)$, then $(x \cdot y) \in$ $m^{\mathcal{I}_{2}}\left(N^{t}\right)$ and $(x \cdot y)=g(x) \cdot g(y)$, where $g$ : $m^{\mathcal{I}_{2}}\left(N^{t}\right) \rightarrow \mathbb{R}$ is defined by $g(x)=\left|\mathcal{I}_{2}-\lim N^{t}(x)\right|$.

Proof. For $\epsilon>0$,

$$
A=\left\{(n, m) \in \mathbb{N} \times \mathbb{N}:\left|N_{n, m}^{t}(x)-g(x)\right|<\epsilon\right\} \in \mathcal{F}\left(\mathcal{I}_{2}\right)
$$

and

$$
B=\left\{(n, m) \in \mathbb{N} \times \mathbb{N}:\left|N_{n, m}^{t}(y)-g(y)\right|<\epsilon\right\} \in \mathcal{F}\left(\mathcal{I}_{2}\right)
$$

where $\epsilon=|x-y|_{*}=\sup _{n, m}\left|N_{n, m}^{t}(x)-N_{n, m}^{t}(y)\right|$.

Now, we have

$$
\begin{aligned}
& \left|N_{n, m}^{t}(x \cdot y)-g(x) g(y)\right| \\
& \quad=\mid N_{n, m}^{t}(x) N_{n, m}^{t}(y)-N_{n, m}^{t}(x) g(y) \\
& +N_{n, m}^{t}(x) g(y)-g(x) g(y) \mid \\
& \leq\left|N_{n, m}^{t}(x)\right|\left|N_{n, m}^{t}(y)-g(y)\right| \\
& +|g(y)|\left|N_{n, m}^{t}(x)-g(x)\right| .
\end{aligned}
$$

As $m^{\mathcal{I}_{2}}\left(N^{t}\right) \subseteq \ell_{\infty}\left(N^{t}\right)$, there exists an $M \in \mathbb{R}$ such that $\left|N_{n, m}^{t}(x)\right|<M$. Therefore, from equation (19), (20) and (21), we have

$$
\begin{aligned}
& \left|N_{n, m}^{t}(x y)-g(x) g(y)\right| \\
& =\left|N_{n, m}^{t}(x) \cdot N_{n, m}^{t}(y)-g(x) g(y)\right| \\
& \leq M_{\epsilon}+|g(y)| \epsilon=\epsilon_{1}, \quad \text { (say) }
\end{aligned}
$$

for all $(n, m) \in A \cap B \in \mathcal{F}\left(\mathcal{I}_{2}\right)$. Hence $(x \cdot y) \in$ $m^{\mathcal{I}_{2}}\left(N^{t}\right)$ and $g(x \cdot y)=g(x) \cdot g(y)$.

\section{Conclusions and Suggestions}

In this, we have introduced a new generalized of Nöurland convergence by using double sequence spaces, these sequences were namely $c_{0}^{\mathcal{I}_{2}}\left(N^{t}\right)$, $c^{\mathcal{I}_{2}}\left(N^{t}\right)$, and $\ell_{\infty}^{\mathcal{I}_{2}}\left(N^{t}\right)$ that is derived by double Nörlund mean. Moreover, we proved some inclusions relation among these spaces. Finally, some properties on these spaces were studied. These new spaces and results related to them furnish new approach to deal with the convergence problems of double sequences occurring in many branches of science and engineering. For future work, we suggest studying these properties by using triple sequence spaces. Furthermore, for application these results can be applied in decision making, intelligence artificial, neutrosophic normed spaces, approximation theorem and so on. 


\section{References:}

[1] C. Boonpok, On $\mathcal{L}^{\star}$-closed sets and some low separation axioms in ideal topological spaces, WSEAS Transactions on Mathematics, 19(2020), 334-342.

[2] C. Boonpok and C. Viriyapong, Almost weak continuity for multifunctions in ideal topological spaces, WSEAS Transactions on Mathematics, 19(2020), 367-372.

[3] C. Boonpok and P. Pue-On, Continuity for multifunctions in ideal topological spaces, WSEAS Transactions on Mathematics, 19(2020), 624631.

[4] P. Das, P. Kostyrko, W. Wilczynski and P. Malik, I and $I^{*}$-convergence of double sequences, Mathematica Slovaca, 58, No. 5 (2008), 605-620.

[5] E. Dundar and B. Altay, $I_{2}$-convergence and $I_{2}$-Cauchy double sequences, Mathematica Acta Scientia, 34B, No. 2 (2013), 343-353.

[6] C. Granados, New notions of triple sequences on ideal spaces in metric spaces, Advances in the Theory of Nonlinear Analysis and its Application, 5(3)(2021), 362-367.

[7] C. Granados, A generalization of the strongly Cesaro ideal convergence through double sequence spaces, International Journal of Applied Mathematics, 34(3)(2021), 525-533.

[8] C. Granados, Convergencia estadística en medida para sucesiones triples de funciones con valores difusos, Rev. Acad. Colomb. Cienc. Ex. Fis. Nat., (2021). https://doi.org/10.18257/raccefyn.1456

[9] C. Granados, A. Dhital, Statistical Convergence of Double Sequences in Neutrosophic Normed Spaces, Neutrosophic Sets and Systems, 42(2021), 333-344.

[10] C. Granados, A.K. Das, B.O. Osu, $M_{\lambda_{m, n, p}}$-statistical convergence for triple sequences, The Journal of Analysis, (2021). https://doi.org/10.1007/s41478-021-00355-0

[11] C. Granados, J. Sanabria, E. Rosas and C. Carpintero, On contra $\Lambda_{I}^{s}$-continuous functions and their applications, Journal of Mathematical and Computational Science, 11(3)(2021), 28342846

[12] C. Granados, Conjuntos pre regulr pc-I-abiertos vía ideales sobre espacios topológicos, Ciencia en Desarrollo, 12(1)(2021), 43-53.
[13] C. Granados, New results on semi-Iconvergence, Transactions of A. Razmadze Mathematical Institute, 175(2)(2021), 199-204.

[14] C. Granados, Pre- $I_{s n}$-open sets and some notions related to pre-I-convergence, Journal of Mathematical Control Science and Applications, 7(1)(2021), 1-9.

[15] G.H. Hardy. divergent series, American Mathematical Soc, 334 (2000) 1-400.

[16] B. Hazarika, A. Alotaibi and S. A. Mohiuddine, Statistical convergence in measure for double sequences of fuzzy-valued functions, Soft Computing, 24 (2020), 6613-6622.

[17] V. Khan, H. Fatima, S. Addullah and K. Alshlool, On paranorm $B V_{\sigma}$ I-convergence double sequence spaces defined by an Orlicz function, Analysis, 37, No 3 (2017), 157-167.

[18] A. Khan, A. Abdullah and K. Alshlool, A study of Nörlund ideal convergent sequence spaces, Yugoslav Journal of Operations Research, 2021.

[19] O. Kisi, Lacunary statistical convergence in measure for double sequences of fuzzy valued functions 2021 (2021), 1-12.

[20] P. Kostyrko, M. Macaj, and T. Salat. Statistical convergence and $\mathcal{I}$-convergence, Real Analysis Exchange, 25(1) (1999) 49.

[21] E. Mursaleen and O. Edely, Statistical convergence of double sequence, J. Math. Anal. Appl., 288 (2003), 223-231.

[22] J. Sanabria, C. Granados, E. Rosas and C. Carpintero, Contra-continuous functions defined through $\Lambda_{I}$-closed sets, WSEAS Transactions on Mathematics 19(2020), 632-638.

[23] A. Yuri, T. Safonova and A. Terekhov, Approximation Functionals and Their Application, WSEAS Transactions on Mathematics, 20(2021), 489-495.

\section{Creative Commons Attribution License 4.0 (Attribution 4.0 International, CC BY 4.0)}

This article is published under the terms of the Creative Commons Attribution License 4.0

https://creativecommons.org/licenses/by/4.0/deed.en US 